\title{
Research on Optimization of Bat Algorithm Based on Gauss Differential Mutation in the Cloud Computing Resources
}

\author{
Xuan Chen \\ Zhejiang Industry Polytechnic College Shaoxing Zhejiang 312000 China \\ 734140999@qq.com
}

\begin{abstract}
Problem of cloud computing resource optimization has always been the research hotspot, as problems of how to satisfy execution speed, average response time and system utilization rate in resource allocation to the greatest extent. The paper first established resource scheduling model of cloud computing, and second, introduced Gaussian differential mutation into individuals of bat algorithm to narrow individual search space. The improved algorithm (GDMBA) has accelerated convergence rate of algorithm and lowered optimization in local. Through comparison with classical test function, it is proved that the algorithm has effectively improved performance of optimizing process. In simulation platform of Cloudsim, GDMBA algorithm has made great improvement in resource scheduling efficiency and task scheduling of cloud computing and effectively improved resource scheduling capacity of cloud computing system.
\end{abstract}

Keywords: bat algorithm, Gauss mutation, difference, cloud computing resources

\section{Introduction}

Since it has been proposed, the concept of cloud computing has always been the hot topic of research. The processing process of cloud computing is to divide the task needs to be processed into several sub-tasks through the Internet, and then return the results to users after calculating and analyzing them through research of the system composed by cloud servers [1]. Cloud computing is the result of distributed computing, parallel computing and grid computing, but different from them, cloud computing is characterized by the commercial model of using according to demands and paying according to demands. Meanwhile, it is influenced by network traffic, network load and network link, etc. There are huge amount of tasks of calculation that need to be processed in cloud computing, and how to make reasonable distribution of tasks and resources has become the index to measure the efficiency of cloud computing.

Literature [2] presents an enhanced fairness allocation algorithm based on the credibility factor CBDRF. The credibility factor was introduced (Credit Factor), for cloud computing in a malicious invasion of punitive behavior resource allocation, to ensure that the platform to other nodes in the resource quotas are not affected. Simulation results show that CBDRF on the premise of ensuring fair distribution enhances guarantees for fairness, effectively ensuring the fairness of resource scheduling and reliability of cloud computing platform. Literature [3] presents a resource scheduling method based on task delays, fully taking into account the schedule cannot be executed immediately, how to configure local computing resources and wait times issues experimental results show that the proposed method can be used to enhance global resource scheduling and local resource scheduling efficiency. Literature [4] proposed has a based on user needs QoS and maximize utility for target of cloud computing resources scheduling model; will meet user budget and most late finished completed time constraints of total utility function as optimization target, then on all task structure decision matrix and return a of processing, and used Lagrange relaxation seeking take property weight vector, to structure out 
eventually of based on multidimensional sex of total utility function; simulation results showed that the algorithm has less of average implementation time and implementation spent, has must of superiority. Literature [5] proposes a cloud environment considered debris of resources with dynamic resource management strategy. Set up fragments of a dynamic resource scheduling model based on cloud computing environment. Simulation experiments show that the resource management policy can effectively fragments the optimization and reorganization of resources, Increase resources to capacity of a successor task. Literature [6] proposed using discrete Particle Swarm Optimization algorithm for load balancing in the cloud computing environment for research, each resource node as a network topology in which each node, established appropriate resource-task assignment model using discrete Particle Swarm Optimization algorithm of resource load balancing. Simulation results show that the algorithm improves resource utilization and load balancing cloud computing resources. Literature [7] proposed an improved artificial Firefly algorithm. Of the algorithm on the Lucifer in value improvement, can prevent convergence quickly, found by Cloudsim simulation platform, under the cloud computing model, the algorithm can reduce the processing task average task completion time, improves the efficiency of tasks dealing with, to the rational allocation of resources. Literature [8] proposes a scheduling model based on chaotic Particle Swarm Optimization algorithm for cloud computing resources. First cloud computing resources as an objective function for load balancing to prevent prematurity, local optimal solutions and other defects appear. Results showed that the algorithm can quickly find the optimal scheduling of cloud computing resources, improve the efficiency of resources utilization, it is practical and feasible. Literature [9] for a cloud computing environment based on improved algorithm SFLA (Shuffled Frog Leaping Algorithm, SFLA) resource scheduling solution. Improvement of individual selection based on QoS values SFLA through CloudSim platform shows that the algorithm is effective. Literature [10] proposed based on chromosome encoding and fitness function of genetic algorithms, simulation results show that the algorithm to the performance and quality of service QoS (Quality of Service) aspects achieved better results, better for large-scale cloud computing resource scheduling of tasks.

Resource allocation in cloud computing involves contents of various aspects: running time of task, costs of task and stability of the task's network chain, so resource distribution in cloud computing is an optimization deployment NP problem of multiple targets. In order to better solve this optimization problem of multiple resources, cuckoo algorithm is proposed in this paper to solve the current resource problem in cloud computing aiming at the search characteristics of cuckoo algorithm and problems in cuckoo algorithm itself. This paper first introduces Gaussian mutation operator to handle the selection of the optimal location of bird nest at each stage, and then adjusts the location of bird nest at different stages through self-adaptive dynamic factors to improve the convergence accuracy of improved algorithm. It has been proved through cloud computing platform that algorithm in this paper improves the efficiency of resource allocation in cloud computing to some extent and reduces the consumption of the network.

\section{Relevant Basic Knowledge}

\subsection{Resource Model in Cloud Computing}

Resource scheduling in cloud computing is actually a problem of balancing and optimizing multiple resources, and a problem of resource planning in its nature. Before constructing cloud computing model in this paper, the following 4 conditions need to be noticed: (1) consider the network load of each node; (2) consider that the amount of tasks in cloud computing are with a certain range, but could not be increased or reduced blindly; (3) time to complete each task; (4) costs to operate nodes in the network.

A six-variable group $G=\{S, T, N, R, E, C\}$ is used to represent the resource scheduling 
model in cloud computing, herein:

(1) $S=\left\{S_{1}, S_{2}, \cdots \cdots S_{m}\right\}$ Refers to $m$ resource sets;

(2) $T=\left\{T_{1}, T_{2}, \cdots \cdots T_{n}\right\}$ Refer to the task set composed of $n$ users

(3) $N=\left\{N_{1}, N_{2} \cdots \cdots N_{z}\right\}$ Refers to $z$ nodes

(4) $R=\left\{R_{11}, R_{12}, \ldots \ldots . R_{x y}\right\}$ Refers to the implementation of task $x$ at node $y$; when $R_{x y}$ is 0 , it refers to that the task $x$ is not at the node $y$, or it refers to implement the task.

(5) $E=\left\{E_{11}, E_{12}, \cdots \cdots E_{x y}\right\}$ Refers to the time of task $x$ on the node $y$.

(6) $C=\left\{C_{11}, C_{12}, \cdots \cdots C_{x y}\right\}$ Refers to the costs of task $x$ on the node $y$.

According to the above analysis, target function of resource allocation in cloud computing in this paper is as shown in formula (1), that is, the node must spend the minimum time and costs. In formula (2), variance is used as the index to measure resource allocation in formula (1).

$$
\begin{aligned}
& \text { min } F_{\varsigma}=\left(f_{\varsigma 1}, f_{\varsigma 2}, \cdots \cdots f_{\zeta k}\right) \\
& f_{\zeta k}=\frac{1}{S} \sum_{t=1}^{s}((E(t)-\overline{E(t)})+(C(t)-\overline{C(t)})) \\
& E(t)=\sum_{i=1}^{T} \sum_{j=1}^{N} E_{i j}(t) \\
& \text { s.t } \\
& \overline{E(t)}=\sum_{i=1}^{T} E_{i j}(t) \\
& \mid C(t)=\sum_{i=1}^{T} \sum_{j=1}^{N} C_{i j}(t) \\
& \overline{C(t)}=\sum_{i=1}^{T} C_{i j}(t)
\end{aligned}
$$

\subsection{Basic Cuckoo Algorithm}

In the nature, in order to find the place to produce eggs, cuckoos adopt a random, and this mainly through the Levy flight paths in stochastic method in the process of candidate generation bird's nest and the adoption of best retention policy updating currently look for nest locations, eventually making the nest location at or near the best solution [11], and Yang put forward a multi-objective algorithms can be adapted to meet multi-objective optimization problems, three ideal cuckoo algorithm:

(1) select a host bird nest at random for the cuckoo $i$, and produce $N$ eggs to represent $N$ target functions respectively.

(2) select a bird nest at random and remain the best host bird nest to the next generation

(3) the probability for host bird to find other eggs is $P$, and if other eggs have been found, choose another bird nest again.

Therefore, based on the three ideal statuses, the update formula for cuckoos to find the path and location of bird nest is as follows:

$x_{i}^{(t+1)}=x_{i}^{(t)}+\alpha \otimes L(\lambda)$

Herein, $x_{i}^{(t+1)}$ refers to the location of bird nest $i$ of the $t$ generation; $\otimes$ is the point-to-point multiplication, $\alpha$ is the pace length and $L(\lambda)$ is the Levy random search path. After the renewal of location, a random number $\mathrm{r}$ is compared with $P$. If $r>P$, 
make change to $x_{i}^{(t+1)}$; otherwise, remain unchanged and remain the location of current bird nest and note it as $x_{i}^{(t+1)} \rightarrow y_{i}^{(t+1)}$.

\subsection{Limitation of Standard Cuckoo Algorithm in Cloud Computing Resources}

In the basic cuckoo algorithm, due to random step cannot be controlled, leading to optimal algorithm for local and slow convergence characteristics and serious effect on precision. Such on easy led to in cloud computing in the node in distribution task of when appeared resources, time and consumption network costs appeared not stable of situation, while easy caused in cloud computing in the cannot makes target function reached minimum value of situation, cuckoo algorithm in evolution of process in the, bird group in the of other cuckoo will attract individual quickly to nest gathered, to led to population diversity reduced, makes algorithm convergence speed greatly reduced even stagnation, on cloud computing in the of resources distribution easy caused cannot get most optimization of resources distribution.

\section{Application of Improved Cuckoo Algorithm in Cloud Computing Resource}

\subsection{Gaussian Mutation Factors}

Gaussian mutation factor can adjust the range of mutation dynamically, which is carried out through coding real numbers. Cuckoos nest location algorithm is randomly chosen, by Gaussian mutation operator to adjust all the nests as a $N P$ population, set up as a dimension of an individual to a variable. Set $x_{i}^{k}$ is the $k$-level evolution of individual $i$, such as shown in equation (4), $\mathrm{min}$ and $\max$ represent the minimum number of maximum evolution and evolution. $x_{i}^{k} \in\left[x_{i}^{\text {min }}, x_{i}^{\max }\right]$

$$
x_{i}^{k}=\left(x_{1}^{k}, x_{2}^{k} \cdots \cdots x_{n}^{k}\right)
$$

Evolution of individuals is as shown in formula (5) and (6). Herein, rand $(k)$ is the random function at the dimension $k$.

$$
x_{i}^{k}(\alpha)= \begin{cases}x_{i}^{k}(\alpha-1)+\Delta\left(x_{i}^{k-1}, t\right) & \operatorname{rand}(k) \in[0,0.5] \\ x_{i}^{k}(\alpha-1)-\Delta\left(x_{i}^{k-1}, x_{i}^{\mathrm{min}}\right) & \operatorname{rand}(k) \in[0.5,1]\end{cases}
$$

Herein, $\Delta(t, y)=y \bullet r^{\lambda\left(1-t / T^{\prime}\right)}$

In formula (5) and (6), $t$ is the current iteration time, $r$ is a random number in $[0,1]$, and $T$ is the total iteration time in the algorithm's evolution. $\lambda$ is the algorithm's adjustment parameter and convergence speed of adjustment algorithm. It can be found from formula (5) that when the iteration time is few, $\lambda(1-t / T)$ tends to get close to 1 , so in the beginning of the algorithm, Gaussian mutation factors can search the optimal location of bird nest at the current period in the entire feasible space. With the increase of $t$ iteration time, $\lambda(1-t / T)$ tends to get close to 0 . Therefore, Gaussian mutation factor searches the optimal solution in the current region.

\subsection{Self-Adaptive Dynamic Factors}

In cuckoo algorithm, due to lack of adaptive mechanisms of search results, less able to deal with global searching capability and optimization accuracy. Introduce self-adaptive 
dynamic factors:

$$
\lambda=\frac{\left|x_{i}-\left(x_{\text {worst }}+x_{\text {best }}\right) / 2\right|}{\sum_{j=1}^{n}\left(x_{i}-x_{j}\right) / n}
$$

Herein, $x_{i}$ refers to the current location of current $i$ bird nest, $x_{w o r s t}$ refers to the poorest status of current bird nest's location, and $x_{\text {best }}$ refers to the optimal status of current bird nest's location. $x$ refers to the location of other bird nest around $x_{i}$. $\sum_{j=1}^{n}\left(x_{i}-x_{j}\right) / n$ refers to the average value of difference between the current bird nest $i$ and other bird nests' location. Therefore, the pace length strategy in bird nest is:

Step $_{i}=\left(\left(\right.\right.$ Step $_{\max }+$ Step $\left.\left._{\min }\right) / 2\right) \bullet \lambda$

From equation (7) and (8) in the self-adaptive dynamic step, when a bird's nest from the Middle average close time, step-less, when far from the average position in the middle of time, step increases. Advantages of Adaptive step size that can be updated according to the results of the previous iteration to iteration moves step, so you can ensure avoiding local optimization, and algorithm optimization accuracy has greatly improved.

\subsection{Introduce Fitness Function}

In order to meet the formula (1) for nodes in the time and cost of spending requirements, so cuckoo algorithms introduced in the fitness function, by selecting the relative fitness of individuals to cloud computing resource scheduling is smaller than the fitness of the individual probabilities for success are. Task scheduling in cloud computing resources, needs to take into account the needs of all child tasks to complete time and expense.

Define time fitness function

$$
f_{\text {time }}(i)=\frac{\sum_{i=1}^{M} \operatorname{Time}(i)}{\text { MaxTime }(i)}
$$

Herein, MaxTime(i) refers to the maximum time to complete all the tasks at the node $i$, and Time (i) refers to the time to complete tasks at the node $i$.

Define the fitness function of costs

$$
f_{\cos t}(i)=\frac{1}{\sum_{i=1}^{M} \operatorname{Cost}(i)}
$$

Herein, Cost $(i)$ refers to the costs to complete task at the node $i$.

In the algorithm of this paper, mainly consider time and costs as constraints of adapted degrees function in the, cloud computing resources utilization more high, if only consider time as single constraints conditions words, all child task run completed of time on will more less, to adapted degrees of value on more big, similarly, only consider costs as single constraints conditions words, all child task run completed by need individual cost more low, its adapted degrees of value on more big. Therefore, in order to better balance the relationship between the two, the Adaptive function defines the following 
$F=\alpha \times f_{\text {time }}(i)+\beta \times f_{\cos t}(i)$

Herein, $\alpha \in[0,1], \beta \in[0,1]$, and $\alpha+\beta=1$

In order to better describe the cloud node in the allotted time and costs, cloud computing nodes and the cuckoo algorithm corresponding to the nodes one by one, select the best individuals in the algorithm that is the best of cloud computing node, in order to better carry out individual choice, we use genetic algorithms.

(1) Operation of Choice

By comparing individuals between the candidate individual $h_{i}^{t}$ and the father node $R_{i}^{t}$ of current individual, operation of choice inherits fine individuals to the next generation, and the rules are as followed:

$x_{i}^{t}=\left\{\begin{array}{l}h_{i}^{t} \\ R_{i}^{t}\end{array}\right.$

(2) Mutation Operation

Set the individual needed to be mutated in current cuckoo as $x_{i}^{t}$, select $N$ individuals in current cuckoo, and produce new individual $g_{i}^{t}$.

$$
g_{i}^{t}=x_{i}^{t}+\kappa \sum_{i=1}^{N} x_{i}^{t}-x_{i+1}^{t}
$$

(3) Crossover Operation

Restructure the individual $g_{i}^{t}$ after mutation operation of current individual and father node $R_{i}^{t}$ to produce candidate individuals.

$$
d_{i}^{t}=\left\{\begin{array}{l}
R_{i r}^{t} \\
g_{i r}^{t}
\end{array}\right.
$$

Herein, is the probability of crossover, and set it less than 1.

\section{Description of Algorithm}

Step 1. Initialize the parameter. Herein, the amount of bird group is $N u m$, iteration amount is $N$, which satisfies that $N<\max$. Initialize the location of individuals in bird nest. Set the minimum pace length is $S$ te $p_{\min }$, and the maximum pace length is $S$ tep $p_{\max }$.

Step 2. For the characteristics of cloud computing resources, cloud computing nodes corresponding to the cuckoo individuals one by one, from the distribution characteristics of cloud computing resources, the cuckoo is divided into $m$ groups $\left(m<\left\lfloor\log _{2} N u m\right\rfloor\right)$.

Step 3. Calculate the solution of a cuckoo's location in a certain subgroup according to formula (3).

Step 4. According to equation (5) and (6) on the same subnet in the Gaussian mutation cuckoo location updates, obtain the new position $x_{i}^{(t+1)}$ of individual at the current location until all the cuckoos in the same group have been updated, and then note them as $\left\{x_{i}^{t} \mid i=1 \cdots n, t=1 \cdots m\right\}$.

Step 5. Introduce formula (7) and (8) according to the updated location in step 4. Optimized the distance between groups. According to selected individuals through (12)-(14) operation to get different cuckoo individuals using (11) nodes are nodes that correspond to these individuals fitness function to calculate the optimal node. 
Step 6. if the iteration time is less than the max, turn to step 2.

Step 7. If the termination condition is met, stop the search for the optimal location; otherwise, turn to Step 2 to continue the optimization.

Step 8. Change the position of the optimal individuals into corresponding resource allocation scheme in cloud computing according to the cuckoo algorithm.

\section{Analysis of Simulation Experimental Results}

In this paper, CloudSim [12] platform is chosen for the test, and the CPU is core i3 and 8GDDR3 while the operation system is Windows Xp. In this paper, distribution of resource in cloud computing is carried out from three aspects.

(1) Comparison with Basic Cuckoo Algorithm (CS)

Set there are 800 virtual tasks and 50 virtual nodes. It can be found by comparing energy consumption of two algorithms that algorithm in this paper consumes more energy in the beginning, mainly because Gaussian mutation and self-adaptive factors are adopted to make the algorithm have larger vibration at first and then tend to be stable later. Compared with the energy vibration during the process of CS algorithm, algorithm in this paper has lower consumption of energy than CS algorithm, which is as shown in Figure 1.

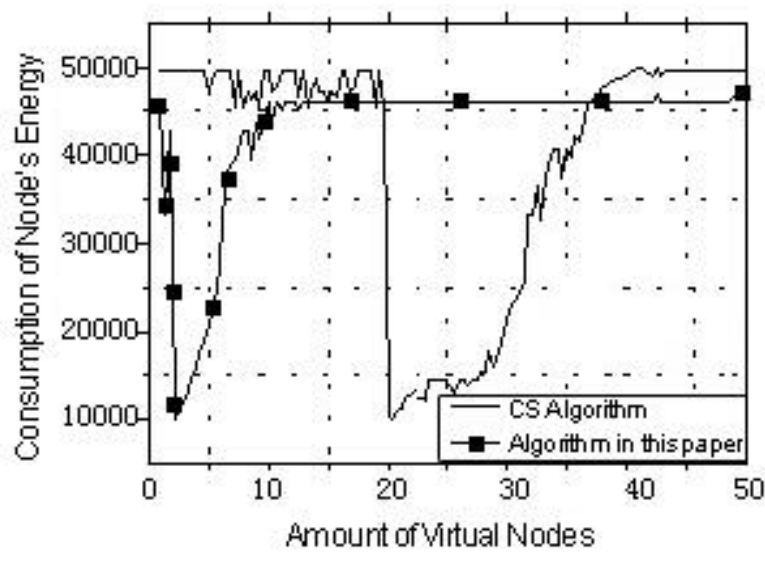

Figure 1. Comparison of Energy Consumption between 2 Resource Loaded Algorithms

(2) Comparison with Algorithm in References

Set there are 800 virtual tasks, 70 virtual nodes and the iteration times is 300 . Compare algorithm in this paper and that in Literature [2], Literature [3] and Literature [6] in the condition of different amount of tasks and virtual nodes in cloud computing model. 


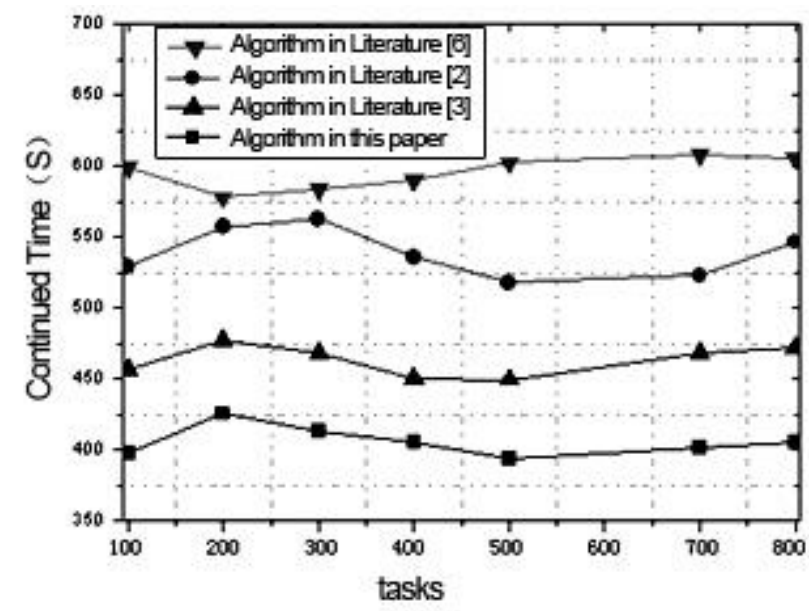

Figure 2. Comparison of Time to Complete Tasks between 4 Resource Loaded Algorithms

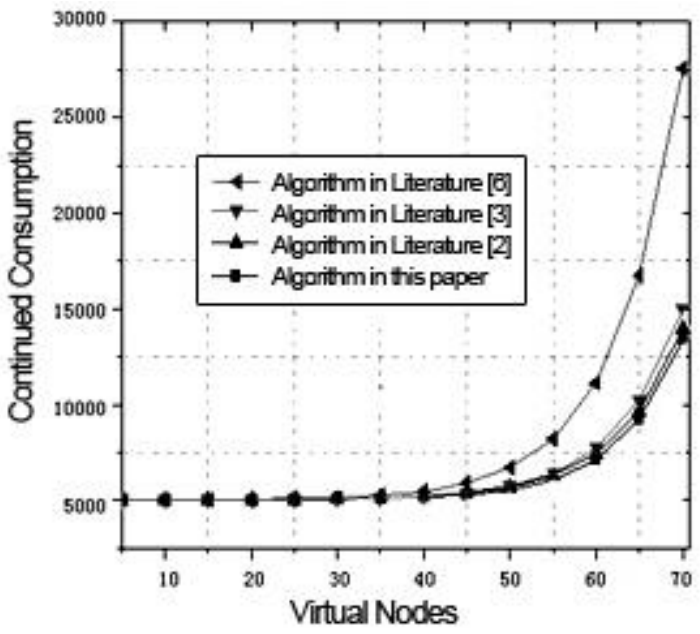

Figure 3. Comparison of Energy Consumption between 4 Resource Loaded Algorithms

It can be found from Figure 2 that this algorithm in terms of resource load time is lower than the other three reference algorithm and is increasing along with the number of tasks and time consuming small range, this algorithm is relatively stable, resource load balancing can better. From the modified algorithm is found in Figure 3 in a cloud computing environment with virtual node network consumption in more energy than the other three references algorithm. Introduction from the above algorithms can be found in the fitness function can effectively balance time and the relationship between costs, so that they can better meet the requirements of cloud computing resource scheduling.

\section{(3) Comparison with Other Intelligent Algorithms}

Select 500 tasks run on a platform in the cloud computing resources is 50, using GA, PSO, FA and the algorithm to distribute the tasks and resources, this algorithm can be found in Figure 4 is different from the other three intelligent algorithm, derived cloud computing resource scheduling performance cost of this task is better solution. From Figure 5, you can find this algorithm differs from the other three algorithms in terms of task completion time for cloud computing resource scheduling performance better programs. 


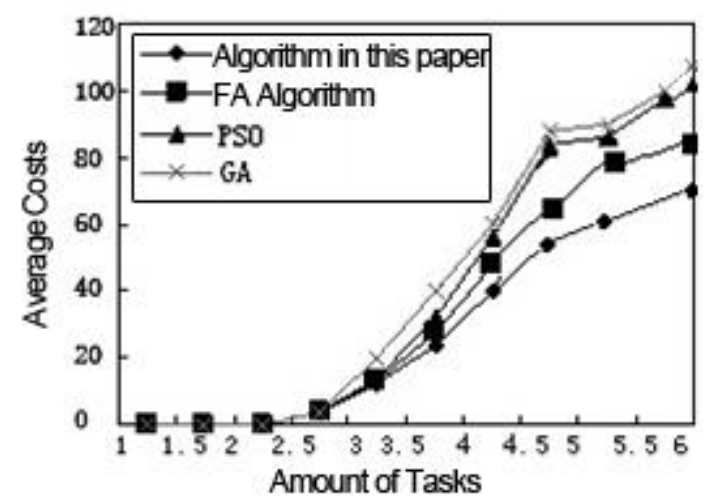

Figure 4. Comparison of Costs to Complete Tasks

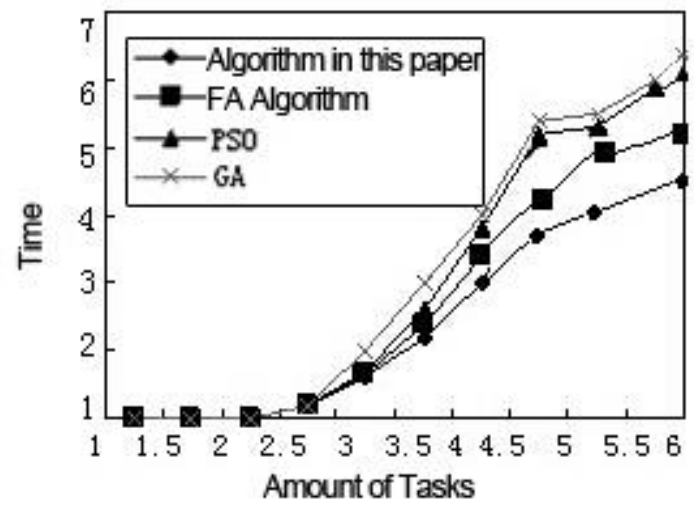

Figure 5. Comparison of Time to Complete Tasks

\section{Conclusion}

The research of cloud computing has always been the focus of research. In this paper, cuckoo algorithm is introduced into resource algorithm in cloud computing. Aiming at fast convergence speed and being easy to have local shocks in cuckoo algorithm, this paper first introduces Gaussian mutation factors to deal with the optimal location selection of bird nest at each stage, and then adjust the selection of bird nest at each stage through self-adaptive dynamic factors so as to improve the convergence accuracy of improved algorithm. Through the balance of self-adaptive function and three operations of genetic algorithm, algorithm in this paper can effectively improve the efficiency of resource allocation in cloud computing, and reduce network consumption. It has been shown through experiment that algorithm in this paper can effective meet the resource allocation model of cloud computing and provide certain value of reference for resource allocation in cloud computing.

\section{References}

[1] C. Kang and Z. Wei-Min, "Cloud Computing: System Instances and Current Research", Journal of Software, vol. 20, no. 5, (2009), pp.1337-1348.

[2] L. Di, "Enhanced fairness-based multi-resource allocation algorithm for cloud computing[J]".Journal of Xidian University, vol. 41, no. 3, (2014), pp.175-181.

[3] Y. Zhao-feng, W. Qi-ming, L Hai-lian, "Research on Resource Scheduling Algorithm of Cloud Computing Based on Improved DAG Diagram and Task Delay[J]", Computer Measurement \& Control , vol. 22, no. 2, (2014), pp. 499-502.

[4] L Bo, L Qing-feng, "Resource Scheduling Model in Cloud Computing Based on QoS and Utility[J]", Computer Measurement \& Control, vol. 22, no. 3, (2014), pp. 826-828. 
[5] W Xiao-yu, "Dynamic resource management mechanism of debris resources in cloud computing[J]" .Journal of Computer Applications, vol. 34, no. 4, (2014), pp. 999-1004.

[6] F Xiao-jing,P Yu, "Load balancing algorithm of DPSO resources in cloud computing environment[J]", "Computer Engineering and Applications", vol. 49, no. 6, (2013), pp. 105-108.

[7] L Li, "Study on improved artificial firefly algorithm in cloud computing resources[J]".Application Research of Computers, vol. 30, no. 8, (2013), pp. 2298-2300.

[8] X Yu, "Research on resource scheduling optimization model based on cloud computing environment[J]".Computer simulation, vol. 30, no. 5, (2013), pp. 362-364.

[9] L Jian-ping,L Xia, "Chen Min-rong.Resource scheduling based on shuffled frog leaping algorithm in cloud computing environment[J]".Computer Engineering and Applications, vol. 48, no. 29, (2012), pp..67-72

[10] L Yu, "Resource scheduling strategy for optimizing genetic algorithm in cloud computing environment[J]" .Journal of Beijing Normal University(Natural Science), vol. 48, no. 4, (2012), pp.378-383.

[11] X YANG, D SUASH, "Cuckoo search via Levy flight[C]//Proceedings of the 2009 World Congress on Nature\&Biologically Inspired Computing.Piscataway”, NJ:IEEE Press,(2009), pp. 210-214.

[12] RN CALHEIROS, R RANJAN, ROSE CAFD, R BUYYA, "CloudSim: a novel framework for modelingand simulation of cloud computing infrastructures and services[EB/OL]”, [2009-93].

\section{Author}

Xuan Chen (1979.03-), male, master, lecturer, research direction: information security, wireless sensor and cloud computing. 